\title{
Gestão da informação na Embrapa Amazônia Oriental: uso relativo versus uso efetivo da literatura técnico-científica agropecuária periódica - 1990-1999
}

Rubenise Gato

Bibliotecária. Mestre em ciência da informação.

E-mail: rubenise@cpatu.embrapa.br

\section{Maria Helena Kurihara}

Supervisora da biblioteca da sede da Empresa Brasileira de Pesquisa

Agropecuária (Embrapa). Mestrada em biblioteconomia - PUCCampinas.

E-mail: Maria.Kurihara@embrapa.br

\section{Lucilda Sousa de Matos}

Bibliotecária

Rosa Melo Dutra

Bibliotecária

Celia Lopes Pereira

Bibliotecária

Isanira Vaz Pereira

Bibliotecária

Silvio Lima Costa

Bibliotecário

\section{Resumo}

Análise bibliométrica de uso da coleção de periódicos, evidenciando o papel fundamental que as bibliotecas da Embrapa desempenham na estrutura organizacional e na estratégia competitiva da empresa. Bibliotecas formam a interface entre a geração e a disseminação do processo de pesquisa, desenvolvimento e inovações ( PD\&I). Resultados apontaram que $77,05 \%$ dos títulos de periódicos utilizados efetivamente encontram-se no Catálogo de Periódicos da Embrapa (CCPE), dos quais 81,83\% pertencem ao Catálogo de Periódicos da Unidade, demonstrando a eficácia do acervo da biblioteca para as atividades de PD\&l na Unidade, no período estudado. Dos títulos estrangeiros adquiridos por compra, 20\% atingiram um indicador de freqüência de uso no período > 10, merecendo priorização de manuteção de assinaturas e processos de preservação.

Palavras-chave

Gestão de coleções; Bibliometria; Informação agropecuária.

Information management at Embrapa Amazonia Oriental: relative versus real usage of agricultural technical-scientific journal literature - 1990-1999

\section{Abstract}

Bibliometric analysis of the journal collection usage, points out the fundamental role played by the Embrapa libraries in the structural organization and in the competitive strategy of the organization. Libraries make up the interface between generation and dissemination of Research, Development and Innovation processes (RD \& I). Results show that $77,05 \%$ of effectively used titles are available through the CCPE - Union Catalog of Embrapa Journals, out of which $81,83 \%$ can be located at the Unit itself, demonstrating the efficacy of the library collection for RD \& I activities in that focused period. Out of the titles effectively bought, $20 \%$ were used $>10$, needing priority in the processes of subscription maintenance and preservation.

Keywords

Collection management; Bibliometrics; Agricultural information.

\section{INTRODUÇÃO}

Os recursos financeiros investidos em assinaturas e/ou acesso a periódicos científicos para suporte à pesquisa agropecuária, na Embrapa, ao longo dos anos, têm sido vultosos por tratar-se da principal fonte que caracteriza a dinâmica da ciência e ser o canal mais relevante para a comunidade científica. Análises de uso do acervo são iniciativas que podem identificar os periódicos mais utilizados e contribuir com informações de apoio para melhoria de processos integrados de gestão e produção de conhecimento organizacional da empresa.

Atualmente a Biblioteca da Embrapa Amazônia Oriental, fundada em 1942 e oriunda do Instituto Agronômico do Norte (IAN) e posteriormente do Instituto de Pesquisas Agropecuárias do Norte (Ipean), incorporada à Embrapa em 1973, conta com um acervo em torno de 32 mil documentos sob vários suportes, além de 325 títulos de acervo raro e uma coleção de periódicos com 3.146 títulos, representando $48 \%$ do Catálogo Coletivo de Periódicos da Embrapa (CCPE).

Desde sua implantação, a biblioteca atende a um público diversificado: pesquisadores, professores, comunidade estudantil de graduação e pós-graduação, técnicos e bibliotecas na região e estudantes de 1 e e 2o graus de escolas públicas e privadas. Nos últimos anos, tem se constituído como base de suporte ao estabelecimento de cursos de pós-graduação nas especialidades das ciências agrárias junto ao MEC, dos quais três foram estabelecidos em contratos com a unidade e instituições de ensino:

- Curso de Mestrado em Agriculturas Familiares e Desenvolvimento Sustentável - Contrato entre Embrapa / UFPA - Núcleo de Agropecuária;

- Curso de Mestrado em Ciência Animal - Contrato entre Embrapa / UFPA - Núcleo de Agropecuária;

- Curso de Doutorado em Sistemas Agroflorestais Contrato entre Embrapa / FCAP. 
Na última década houve severas limitações de recursos financeiros para investimentos na manutenção e atualização do acervo, especialmente de periódicos e, embora tenha se registrado um esforço para a manutenção de títulos considerados essenciais às atividades de pesquisa desenvolvidas pela empresa, muitos títulos foram suprimidos, outros deixaram de ser adquiridos e ainda outros pararam por descontinuidade de edição, resultando em coleções atuais bastante incompletas e descontinuadas.

O estado atual da coleção de periódicos, do ponto de vista da preservação física, ao longo de 62 anos de existência da biblioteca em relação à completeza e manutenção, provocou a necessidade de realização de estudo de avaliação de uso, no contexto da gestão da informação, visando à eficácia do acervo para as atividades de P\&DI, bem como a identificação dos periódicos de uso efetivo considerados fundamentais para manutenção de suas assinaturas, permanência no acervo e priorização para processos de encadernação.

A gestão da informação é a habilidade de selecionar, de um conjunto de informação disponível, aquela relevante e pertinente para uma tomada de decisão, que pode ser organizada e estruturada sob vários artefatos (Braga, 2004).

Os estudos e pesquisas sobre uso de coleções de periódicos abordam métodos variados: análises quantitativas, qualitativas e inclusão de opiniões de usuários. Os objetivos dos estudos invariavelmente visam a verificar o grau de eficácia dos acervos para subsidiar o estabelecimento de políticas de seleção, aquisição, descarte e encadernação, bem como para medir o uso da literatura periódica (Mueller, 1991; Mueller, 1992b; Lancaster, 1996).

Para organizar e estruturar a informação sob a abordagem da inteligência competitiva, gestão do conhecimento e gestão da informação, a ciência da informação tem na bibliometria uma ferramenta essencial.

Bibliometria é definida por Pritchard em 1969, citado por Pinheiro (1982) como "a aplicação de métodos matemáticos para livros e outros meios de comunicação”. Atualmente tem sido aplicada para o tratamento quantitativo da informação registrada sob qualquer suporte. Suas leis, princípios e técnicas têm sido utilizadas por diferentes áreas como forma de reduzir/tratar/ analisar massas de documentos e torná-las passíveis de serem utilizadas como insumo para tomada de decisões estratégicas, políticas, econômicas, entre outras.
Tague-Sutcliffe, citado por Macias-Chapula (1998), aponta a bibliometria como "estudo dos aspectos quantitativos da produção, disseminação e uso da informação registrada”.

Este trabalho compreende parte do projeto em andamento "Desenvolvimento de modelo para mapeamento das tecnologias geradas pela Embrapa na Amazônia: levantamento da informação e aplicações bibliométricas". O objetivo dessa etapa, além de avaliar o uso da coleção de periódicos do acervo da biblioteca no período 1990-1999, pretende encontrar um método que possa ser empregado sistematicamente como monitoramento de uso dos periódicos na unidade.

\section{OBJETIVOS}

Visando a contribuir para ações no processo de gestão de informação e do conhecimento no centro, a análise objetivou identificar o uso da coleção de periódicos no período de 10 anos, tendo como limite temporal 19901999. Especificamente buscou-se:

- definir e estudar, comparativamente, o uso relativo e o uso efetivo dos periódicos solicitados ao serviço de empréstimo, comutação bibliográfica (Comut) e dos títulos citados na produção técnico-científica da unidade;

- identificar os periódicos científicos de uso efetivo considerados fundamentais para manutenção de suas assinaturas, permanência no acervo e priorização para processos de encadernação.

\section{METODOLOGIA}

Para efeito da análise nas variantes de uso relativo e efetivo, foram consideradas duas situações:

- o fato de a biblioteca possuir um acervo especializado e direcionado a suprir as necessidades de informação de pesquisadores da Embrapa Amazônia Oriental para os processos de P\&DI;

- o fato de a biblioteca também atender a vários usuários externos representados por pesquisadores de outras instituições congêneres, professores, estudantes de graduação e pós-graduação, entre outros.

Essas situações foram determinantes para a adoção de um método de análise que proporcionasse um resultado mais próximo possível da realidade de uso pela comunidade técnico-científica da unidade, considerandose como uso relativo o uso de periódicos solicitados por empréstimos e uso efetivo o uso de periódicos citados na produção técnico-científica dos pesquisadores da unidade, 
Gestão da informação na Embrapa Amazônia Oriental: uso relativo versus uso efetivo da literatura...

tendo em vista que uma análise de dados do serviço de empréstimo pode se converter em apenas um indicador relativo de uso.

A análise foi baseada na Lei de Bradford, uma das leis e técnicas da bibliometria que, embora concebida para analisar a produtividade de periódicos científicos, tem sido empregada em outras abordagens com a finalidade de obter indicadores para diversas ações de gerenciamento da informação, cujo enunciado, citado por Pinheiro (1983) é: "se os periódicos forem ordenados em ordem de produtividade decrescente de artigos sobre um determinado assunto, poderão ser distribuídos num núcleo de periódicos mais particularmente devotados a esse assunto e em diversos grupos ou zonas contendo o mesmo número de artigos que o núcleo, sempre que o número de periódicos e das zonas sucessivas for igual a 1:n:n²..." Para ilustrar a lei, é utilizada a formulação gráfica semilogarítimica, cuja curva tem forma de "S" e é conhecida como gráfico "Bradford-Zipf".

A coleta de dados foi realizada no período de 2000-2001, com base nos seguintes critérios:

- títulos de periódicos somente adquiridos por compra, utilizados no serviço de empréstimo no período de 1990. 1995 em consulta manual nas fichas de controle de empréstimo em cada fascículo;

- títulos de periódicos adquiridos por compra, doação e permuta, solicitados no serviço de empréstimo no período de 1996-1999, processo automatizado, utilizando relatório do aplicativo Ainfo*;

- títulos de periódicos utilizados no serviço de atendimento à comutação bibliográfica no período de 1998. 1999, por atender especificamente usuários do SNPA (registrados em formulários de atendimento e e-mails);

- títulos de periódicos utilizados no serviço de atendimento ao Comut, tendo em vista a biblioteca caracterizar-se como biblioteca-base do programa no período de 1998-1999, por atender a usuários diversos (registrados no formulário do sistema on-line);

- títulos de periódicos citados nos trabalhos publicados no período de 1990-1999. Excluíram-se trabalhos que não relacionavam referências bibliográficas consultadas, entre os quais: Série recomendações básicas, pesquisas em andamento, comunicado técnico e resumos em anais de congressos e correlatos;

\footnotetext{
* Sistema de automação de bibliotecas e gerenciamento de informação técnico-científica. Software desenvolvido pela Embrapa Informática Agropecuária.
}

- contemplar como produção técnico-científica toda aquela editada pelas unidades descentralizadas da Embrapa (UD), antecessoras da Embrapa Amazônia Oriental (Ex-Uepae de Altamira, Ex-Uepae de Belém, Ex-Cpatu ) e de autoria ou co-autoria de pesquisadores lotados nestas UDs, publicadas por outros editores e/ou organismos editoriais;

- levantar a produção editorial da Unidade, no período de 1990-1999, indexada na Base Acervo Documental da Unidade, considerando-se todos os tipos de documentos de acordo com as linhas editoriais: linha técnicocientífica ( veiculados em periódicos nacionais e estrangeiros); linha transferência de tecnologia (boletim de pesquisa, circular técnica, documentos) e documentos caracterizados como publicações avulsas, entre as quais: capítulos, parte e/ou seções de livros, artigos, entre outros, com autoria e/ou co-autoria de pesquisadores da unidade, publicados por outros editores e/ou instituições editoriais;

- conferir os títulos de periódicos identificados nos documentos referentes à produção técnico-científica da unidade, no Ulrich's Periodicals Directory, edição de 1990, disponível na biblioteca, para evitar contagem duplicada do mesmo título de periódico, considerando-se mudanças de títulos, títulos iguais com procedência editorial diferente, entre outros;

- considerar os títulos exatamente como se apresentaram, sem contemplar as eventuais mudanças ocorridas.

O quantitativo de periódicos considerados para a análise foi definido pela totalidade dos títulos identificados tanto dos utilizados nos serviços de empréstimo, comutação bibliográfica e Comut (618 títulos), quanto dos títulos citados na produção técnico-científica dos pesquisadores da unidade (824) no período 1990-1999, correspondendo a 1.442 títulos.

A organização dos dados como requisitos para a aplicação das técnicas e emprego da Lei de Bradford contemplou a seqüência:

- digitação dos títulos em planilhas usando-se o programa Excel da Microsoft;

- disposição em ordem alfabética de títulos;

- ordenação por freqüência de empréstimo no período de 1990-1999;

- ordenação por freqüência de citações no mesmo período. 
A ordenação alfabética incluiu os artigos dos títulos, na expectativa de utilizá-los como elemento identificador, no caso de possíveis títulos iguais com procedências diferentes.

\section{RESULTADOS}

Utilizando-se a mesma lógica da Lei de Bradford, em que a zona inicial corresponde à concentração e às subseqüentes de produtividade decrescente, para a análise de uso da literatura periódica foi considerada a zona inicial como correspondente à maior concentração de empréstimo / citação, seguida das demais até a de menor concentração, ou seja, a zona que demonstra a dispersão de uso dos periódicos.

Análise de periódicos utilizados nos serviços de empréstimo, comutação bibliográfica e programa Comut*

$\mathrm{Na}$ distribuição de acordo com a Lei de Bradford (tabela 1), os títulos foram arranjados por ordem decrescente do numero de empréstimo. A coluna $\mathbf{P}$ indica o número de títulos de periódicos. A coluna $\mathrm{E}$ o número de vezes que o periódico foi emprestado. A coluna PxE é o resultado da coluna $\mathbf{P}$ multiplicado pela coluna $\mathbf{E}$ e revela a freqüência de empréstimo, a coluna $\boldsymbol{\Sigma}$ P o somatório dos periódicos emprestados e a coluna $\boldsymbol{\Sigma} \mathbf{P x E}$ o somatório dos empréstimos.

$\mathrm{Na}$ Divisão Máxima de Zonas, de acordo com os critérios de aplicação da lei, estabeleceu-se margem de 10\% para mais e para menos como limites para enquadramento de zonas subseqüentes. Procurou-se alcançar o máximo de zonas possível, encontrando-se sete zonas, conforme demonstra a tabela 2 , a seguir.

Pela distribuição de comportamento segundo Bradford, como indica a tabela 2 , cada uma das sete zonas concentra aproximadamente $14.0 \%$ do total dos empréstimos - mas em relação ao número de periódicos a distribuição no Núcleo (Z1) e nas zonas sucessivas é de rentabilidade decrescente. As quatro primeiras zonas concentram mais da metade (56.7\%) dos empréstimos e menos de $10 \%$ dos periódicos. A partir da $4^{\mathrm{a}}$ Zona, pode-se observar que para cada uma delas o percentual de empréstimos continua na ordem de $14 \%$, mas o número de periódicos quase dobra nas últimas três zonas. A dispersão na última zona (7) é bem grande - aí estão 2/3 dos periódicos e os mesmos $14 \%$ dos empréstimos.

* O atendimento à comutação bibliográfica e Comut não registra empréstimo para os procedimentos que os serviços exigem; porém, para facilitar a análise, os títulos foram incluídos como empréstimo.
TABELA 1

Periódicos $(\mathrm{P})$ por ordem decrescente de empréstimo (E) da biblioteca, no período de 1990-1999, segundo a distribuição de Bradford. Embrapa Amazônia Oriental, Belém, 2002

\begin{tabular}{|c|c|c|c|c|}
\hline $\mathrm{P}$ & $\mathrm{E}$ & P X E & $\Sigma P$ & $\Sigma \mathrm{P} \times \mathrm{E}$ \\
\hline 1 & 200 & 200 & 1 & 200 \\
\hline 1 & 180 & 180 & 2 & 380 \\
\hline 1 & 168 & 168 & 3 & 548 \\
\hline 1 & 165 & 165 & 4 & 713 \\
\hline 1 & 144 & 144 & 5 & 857 \\
\hline 1 & 134 & 134 & 6 & 991 \\
\hline 1 & 131 & 131 & 7 & 1112 \\
\hline 1 & 124 & 124 & 8 & 1246 \\
\hline 1 & 107 & 107 & 9 & 1353 \\
\hline 1 & 92 & 92 & 10 & 1445 \\
\hline 1 & 71 & 71 & 11 & 1516 \\
\hline 1 & 69 & 69 & 12 & 1585 \\
\hline 1 & 67 & 67 & 13 & 1652 \\
\hline 2 & 57 & 114 & 15 & 1766 \\
\hline 1 & 56 & 56 & 16 & 1822 \\
\hline 1 & 53 & 53 & 17 & 1875 \\
\hline 1 & 52 & 52 & 18 & 1927 \\
\hline 1 & 51 & 51 & 19 & 1978 \\
\hline 1 & 50 & 50 & 20 & 2028 \\
\hline 1 & 49 & 49 & 21 & 2077 \\
\hline 1 & 48 & 48 & 22 & 2125 \\
\hline 4 & 45 & 180 & 26 & 2305 \\
\hline 2 & 44 & 88 & 28 & 2393 \\
\hline 1 & 43 & 43 & 29 & 2436 \\
\hline 1 & 42 & 42 & 30 & 2478 \\
\hline 1 & 39 & 39 & 31 & 2517 \\
\hline 2 & 38 & 76 & 33 & 2593 \\
\hline 1 & 37 & 37 & 34 & 2630 \\
\hline 1 & 36 & 36 & 35 & 2666 \\
\hline 2 & 35 & 70 & 37 & 2736 \\
\hline 2 & 34 & 68 & 39 & 2804 \\
\hline 1 & 33 & 33 & 40 & 2837 \\
\hline 3 & 32 & 96 & 43 & 2933 \\
\hline 4 & 31 & 124 & 47 & 3057 \\
\hline 3 & 30 & 90 & 50 & 3147 \\
\hline 2 & 29 & 58 & 52 & 3205 \\
\hline 1 & 28 & 28 & 53 & 3233 \\
\hline 1 & 27 & 27 & 54 & 3260 \\
\hline 1 & 26 & 26 & 55 & 3286 \\
\hline 5 & 25 & 125 & 60 & 3411 \\
\hline 1 & 23 & 23 & 61 & 3434 \\
\hline 2 & 22 & 44 & 63 & 3478 \\
\hline 3 & 21 & 63 & 66 & 3541 \\
\hline 3 & 20 & 60 & 69 & 3601 \\
\hline 6 & 19 & 114 & 75 & 3715 \\
\hline 6 & 18 & 108 & 81 & 3823 \\
\hline 8 & 17 & 136 & 89 & 3959 \\
\hline 7 & 16 & 112 & 96 & 4071 \\
\hline 4 & 15 & 60 & 100 & 4131 \\
\hline 14 & 14 & 196 & 114 & 4327 \\
\hline 10 & 13 & 130 & 124 & 4457 \\
\hline 5 & 12 & 60 & 129 & 4517 \\
\hline 8 & 11 & 88 & 137 & 4605 \\
\hline 13 & 10 & 130 & 150 & 4735 \\
\hline 9 & 9 & 81 & 159 & 4816 \\
\hline 8 & 8 & 64 & 167 & 4880 \\
\hline 20 & 7 & 140 & 187 & 5020 \\
\hline 30 & 6 & 180 & 217 & 5200 \\
\hline 30 & 5 & 150 & 247 & 5350 \\
\hline 36 & 4 & 144 & 283 & 5494 \\
\hline 39 & 3 & 117 & 322 & 5611 \\
\hline 112 & 2 & 224 & 434 & 5835 \\
\hline 184 & 1 & 184 & 618 & 6019 \\
\hline
\end{tabular}


Gestão da informação na Embrapa Amazônia Oriental: uso relativo versus uso efetivo da literatura...

\section{TABELA 2}

Zona de Divisão máxima de empréstimos (E) dos periódicos (P) no período de 1990-1999, de acordo com a Lei da Bradford. Embrapa Amazônia Oriental. Belém, 2002

\begin{tabular}{crrrrrrrrr}
\hline ZONA & $\mathrm{E}$ & $\boldsymbol{\Sigma} \mathrm{E}$ & $\% \mathrm{E}$ & $\boldsymbol{\Sigma} \% \mathrm{E}$ & $\mathrm{P}$ & $\boldsymbol{\Sigma} \mathrm{P}$ & $\% \mathrm{P}$ & $\boldsymbol{\Sigma} \% \mathrm{P}$ & $\mathrm{mB}$ \\
\hline 1 & 857 & 857 & 14.2 & 14.2 & 5 & 5 & 0.8 & 0.8 & - \\
2 & 852 & 1709 & 14.2 & 28.4 & 9 & 14 & 1.5 & 2.3 & 1.8 \\
3 & 846 & 2555 & 14.1 & 42.5 & 18 & 32 & 2.9 & 5.2 & 2.0 \\
4 & 856 & 3411 & 14.2 & 56.7 & 25 & 60 & 4.5 & 9.7 & 1.6 \\
5 & 846 & 4257 & 14.1 & 70.8 & 49 & 109 & 7,9 & 17.6 & 1.8 \\
6 & 865 & 5122 & 14.4 & 85.2 & 95 & 204 & 15.4 & 33.0 & 1.9 \\
7 & 897 & 6019 & 14.9 & 100.1 & 414 & 618 & 66.9 & 99.9 & 4.4
\end{tabular}

$\mathrm{mB}=13.5 / 6=2.3$

\section{FIGURA 1}

Distribuição de periódicos do empréstimo no período de 1990-1999, de acordo com a ilustração de Bradford. Embrapa Amazônia Oriental. Belém, 2002

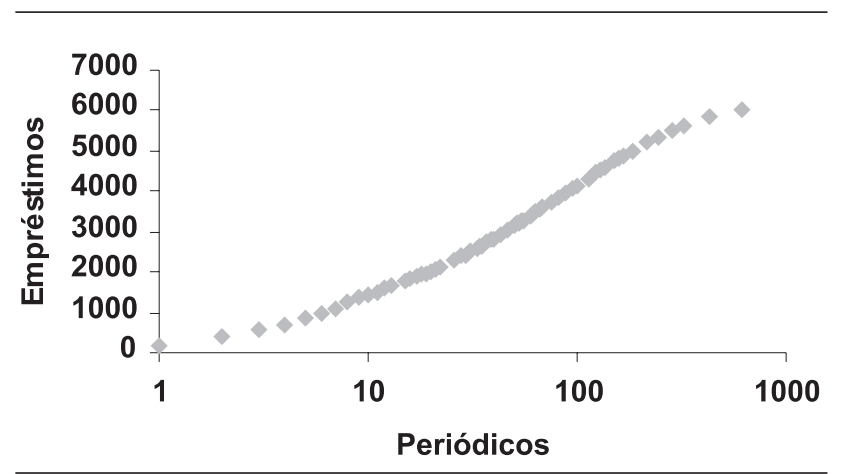

O multiplicador de Bradford foi "pressionado" pelo número de periódicos da última zona. Sem a zona 7, o multiplicador seria 1.8 e não 2.3 como é o caso. Pode-se observar que quadriplicou o número de periódicos da zona 6 (95) para a zona 7 (414).

Conforme demonstrado, do total de 618 títulos de periódicos analisados, 60 títulos atenderam a 56.7\% dos empréstimos realizados pela biblioteca, incluindo empréstimos no balcão e utilizados para atendimento dos serviços de comutação bibliográfica e Programa Comut, cuja distribuição pode ser observada na figura 1 , indicando a maior concentração e dispersão de uso dos títulos.

Análise dos periódicos citados na produção técnico-científica no período de 1990-1999, indexada na Base Acervo Documental (Aplicativo Ainfo) da Unidade.

Na distribuição de acordo com a Lei de Bradford (tabela 3) os títulos foram arranjados por ordem decrescente do número de citações. A coluna $\mathbf{P}$ indica o número de títulos de periódicos. A coluna $\mathrm{C}$ o número de vezes que o periódico foi
TABELA 3

Periódicos $(\mathrm{P})$ por ordem decrescente de citação $(\mathrm{C})$ na produção técnico-científica no período de 1990 1999, segundo a distribuição de Bradford. Embrapa Amazônia Oriental, Belém, 2001

\begin{tabular}{|c|c|c|c|c|}
\hline $\mathrm{P}$ & $\mathrm{C}$ & P X C & $\Sigma \mathrm{P}$ & $\Sigma \mathrm{P} \times \mathrm{C}$ \\
\hline 1 & 191 & 191 & 1 & 191 \\
\hline 1 & 132 & 132 & 2 & 323 \\
\hline 1 & 114 & 114 & 3 & 437 \\
\hline 1 & 67 & 87 & 4 & 524 \\
\hline 1 & 84 & 84 & 5 & 608 \\
\hline 1 & 70 & 70 & 6 & 678 \\
\hline 1 & 68 & 68 & 7 & 746 \\
\hline 1 & 65 & 65 & 8 & 811 \\
\hline 1 & 58 & 58 & 9 & 869 \\
\hline 1 & 57 & 57 & 10 & 926 \\
\hline 1 & 52 & 52 & 11 & 978 \\
\hline 1 & 50 & 50 & 12 & 1028 \\
\hline 3 & 46 & 138 & 15 & 1166 \\
\hline 1 & 44 & 44 & 16 & 1210 \\
\hline 4 & 42 & 168 & 20 & 1378 \\
\hline 2 & 39 & 78 & 22 & 1456 \\
\hline 3 & 38 & 114 & 25 & 1570 \\
\hline 1 & 37 & 37 & 26 & 1607 \\
\hline 5 & 36 & 180 & 31 & 1787 \\
\hline 1 & 35 & 35 & 32 & 1822 \\
\hline 1 & 34 & 34 & 33 & 1856 \\
\hline 3 & 33 & 99 & 36 & 1955 \\
\hline 1 & 30 & 30 & 37 & 1985 \\
\hline 1 & 29 & 29 & 38 & 2014 \\
\hline 1 & 28 & 28 & 39 & 2042 \\
\hline 1 & 27 & 27 & 40 & 2069 \\
\hline 3 & 26 & 78 & 43 & 2147 \\
\hline 2 & 25 & 50 & 45 & 2197 \\
\hline 3 & 24 & 72 & 48 & 2269 \\
\hline 1 & 23 & 23 & 49 & 2292 \\
\hline 1 & 22 & 22 & 50 & 2314 \\
\hline 3 & 21 & 63 & 53 & 2377 \\
\hline 5 & 20 & 100 & 58 & 2477 \\
\hline 6 & 18 & 108 & 64 & 2585 \\
\hline 1 & 17 & 17 & 65 & 2602 \\
\hline 6 & 16 & 96 & 71 & 2698 \\
\hline 2 & 15 & 30 & 73 & 2728 \\
\hline 5 & 13 & 65 & 78 & 2793 \\
\hline 6 & 12 & 72 & 84 & 2865 \\
\hline 7 & 11 & 77 & 91 & 2942 \\
\hline 9 & 10 & 90 & 100 & 3032 \\
\hline 14 & 9 & 126 & 114 & 3158 \\
\hline 17 & 8 & 136 & 131 & 3294 \\
\hline 12 & 7 & 84 & 143 & 3378 \\
\hline 31 & 6 & 186 & 174 & 3564 \\
\hline 22 & 5 & 110 & 196 & 3674 \\
\hline 50 & 4 & 200 & 246 & 3874 \\
\hline 57 & 3 & 171 & 303 & 4045 \\
\hline 167 & 2 & 334 & 470 & 4379 \\
\hline 354 & 1 & 354 & 824 & 4733 \\
\hline
\end{tabular}

citado. A coluna PxC é o resultado da coluna P multiplicado pela coluna $\mathbf{C}$ e revela a freqüência de citações, a coluna $\mathbf{\Sigma} \mathbf{P}$ O somatório dos periódicos citados e a coluna $\mathbf{\Sigma P x} \mathbf{C}_{\text {o somatório }}$ das citações.

Para a divisão máxima de zonas, de acordo com os critérios de aplicação da lei, estabeleceu-se margem de $10 \%$ para mais e para menos como limites para enquadramento de zonas subseqüentes. Nos 824 títulos 
considerados para a análise, de acordo com os procedimentos da lei, buscou-se alcançar o máximo de zonas possível, encontrando-se sete zonas, conforme demonstra a tabela 4 .

Pelo comportamento de Bradford, cada uma das sete zonas concentra $\approx 14.0 \%$ do total das citações; entretanto, em relação ao número de periódicos, a distribuição no núcleo (Z1) e nas zonas sucessivas é de rentabilidade decrescente. As quatro primeiras zonas concentram metade das citações $(\boldsymbol{\Sigma} \% \mathrm{C}) \mathbf{5 7 . 1 \%}$, cobertas por $8,7 \%$ dos periódicos analisados. As zonas seis e sete, juntas, cobriram 28,4\% das citações, menor concentração, porém dispersos em $77.9 \%$ dos periódicos. A distribuição indicando maior concentração e dispersão dos periódicos citados pode ser observada na figura 2 .

Considerando que, tanto na análise dos periódicos utilizados no serviço de empréstimo quanto na análise dos periódicos citados, a maior concentração coincidiu nas quatro primeiras zonas de divisão máxima de Bradford, foi elaborada uma matriz de coincidências de freqüência dos periódicos enquadrados nas quatro primeiras zonas, encontrando-se 116 títulos, com 49\% coincidentes em empréstimo e citações, dos quais $67 \%$ são de origem estrangeira e 33\% nacionais. Do conjunto desses títulos, 81,73\% foram citados na produção técnicocientífica da unidade no período.

Neste conjunto foram identificados periódicos que não possuem características de periódicos científicos. Entretanto, o uso desse tipo de literatura emergiu pelo fato de, atualmente, encontrar-se com mais freqüência notícias em cadernos especiais ou reportagens sobre determinados temas abordando informações de C\&T.

Os 49\% de títulos coincidentes tanto espelham o fato de a análise de empréstimo haver considerado todas as categorias de usuários da biblioteca ( professores, alunos de graduação e pós-graduação, pesquisadores, entre outros) quanto a análise de citação haver contemplado somente os usuários do quadro técnico-científico da unidade.

Justapondo-se os títulos de periódicos identificados na análise de empréstimo com os da análise de citação, foram realizadas conferências de títulos no Ulrich's e ajustes nas nomenclaturas (uma vez que as análises foram efetuadas considerando-se os títulos como se apresentaram no levantamento dos dados), encontrando-se um total de 1.129 títulos. Destes 870, representando 77,05\%, encontram-se disponíveis na Embrapa, entre acervos, biblioteca virtual Embrapa e bases de dados, contra 259 representando $22,94 \%$ citados na produção técnico-

\section{TABELA 4}

Zona de divisão máxima de citações (C) dos periódicos ( $\mathrm{P}$ ) na produção técnico-científica, no período de 1990-1999, segundo Bradford . Embrapa Amazônia Oriental. Belém, 2002

\begin{tabular}{crrrrrrrrr}
\hline ZONA & $\mathrm{C}$ & $\boldsymbol{\Sigma} \mathrm{C}$ & $\% \mathrm{C}$ & $\boldsymbol{\Sigma} \% \mathrm{C}$ & $\mathrm{P}$ & $\boldsymbol{\Sigma} \mathrm{P}$ & $\% \mathrm{P}$ & $\boldsymbol{\Sigma} \% \mathrm{P}$ & $\mathrm{mB}$ \\
\hline 1 & 678 & 678 & 14.3 & 14.3 & 6 & 6 & 0.7 & 0.7 & - \\
2 & 680 & 1358 & 14.3 & 28.6 & 14 & 20 & 1.6 & 2.3 & 2.3 \\
3 & 677 & 2035 & 14.3 & 42.9 & 19 & 39 & 2.3 & 4.6 & 1.3 \\
4 & 676 & 2711 & 14.2 & 57.1 & 34 & 73 & 4.1 & 8.7 & 1.7 \\
5 & 675 & 3386 & 14.2 & 71.3 & 101 & 174 & 12.2 & 20.9 & 2.9 \\
6 & 674 & 4060 & 14.2 & 85.5 & 296 & 470 & 35.9 & 56.8 & 2.9 \\
7 & 673 & 4733 & 14.2 & 99.7 & 354 & 824 & 42.9 & 99.7 & 1.1 \\
\hline $\mathrm{mB}=$ & $\mathbf{2 . 0}$ & & & & & & & &
\end{tabular}

FIGURA 2

Distribuição de periódicos citados no período de 1990-1999, de acordo com a ilustração de Bradford. Embrapa Amazônia Oriental. Belém, 2002

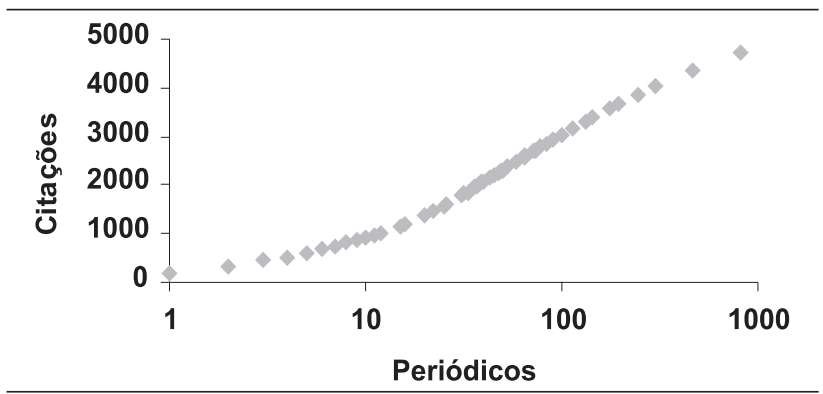

científica não existentes no CCPE, dos quais grande parte encontra-se nas zonas sete de maior dispersão.

Dos títulos nacionais e estrangeiros emprestados/citados disponíveis na empresa, (712), representando 81,83\%, foram utilizados do acervo da biblioteca da unidade, demonstrando a eficácia do acervo para as atividades de P\&DI no período, contra (102 títulos) representando 12,09\% (artigos) solicitados via comutação bibliográfica/ Programa Comut.

Dos títulos estrangeiros adquiridos por compra identificados na análise da tabela 5, a seguir, $11 \%$ atingiram freqüência de uso $>20 ; 9 \%>10 ; 46 \%<10$ e $35 \%<2$. Dessa forma, pode-se indicar que 59 títulos são considerados fundamentais para manutenção no acervo e essenciais para suporte às atividades de P\&DI na Unidade.

Comparando-se com o acervo total 3.146 títulos na base catálogo de periódicos da unidade (CPU), em 10 anos foram utilizados $22,63 \%$ do acervo da biblioteca, e $77,36 \%$ constituiu-se no período como memória científica de interesse preservada em várias áreas do conhecimento.

Na composição das zonas de divisão máxima de Bradford dos periódicos emprestados e citados, observou-se que, 
Gestão da informação na Embrapa Amazônia Oriental: uso relativo versus uso efetivo da literatura...

nas duas primeiras zonas (Z1) de maior concentração, houve uma tendência de agrupar periódicos com assuntos/temas mais gerais, enquanto os periódicos com assuntos/temas mais específicos encontram-se distribuídos nas zonas cinco e seis subseqüentes, ensejando um indicador de que periódicos especializados nas diversas áreas do conhecimento podem influenciar a freqüência de empréstimos e citações.

No que concerne às zonas sete, que apresentam expressiva dispersão tanto na análise de empréstimo quanto na de citações, a julgar pelos assuntos cobertos pelos periódicos, dadas suas especializações, é factível serem identificadas áreas e/ou temas emergentes com assuntos com menor concentração de estudos, além de vários fatores que podem ser intervenientes nesta dispersão, tais como: assuntos/temas ainda pouco explorados em termos de pesquisas na unidade, diversidade de temas/assuntos pesquisados na unidade pelas características de centro ecorregional e quantitativo desbalanceado de especialistas em relação à abrangência das pesquisas.

Em termos de desbalanceamento de competências para execução da atividade de pesquisa, isto pode ser observado na análise realizada por Penteado \& Quonian (2001). Por exemplo, se em determinada área a unidade dispõe de uma equipe de pesquisadores concentrando esforços em determinada área, a probabilidade de utilização de periódicos especializados nessa área tende a ser maior - seja no empréstimo ou na citação. No caso da unidade, provavelmente este indicador esteja relacionado com as equipes de pesquisadores e/ou suas especialidades, bem como com a programação de pesquisa da unidade no período estudado.

Comparando-se os títulos estrangeiros disponíveis citados e emprestados com o quantitativo das listas consideradas básicas, resultante da avaliação de títulos apontados como essenciais ao Programa de Aquisição Planificada de Periódicos para apoiar a pesquisa e o ensino de profissões agroindustriais nos cursos de pós-graduação no Brasil, realizada por Mueller (1992a) e Mueller $\left(1992 b^{*}\right)$, dos títulos indicados pelos professores/ pesquisadores das instituições de ensino e pesquisa identificou-se o seguinte:

\footnotetext{
* MUELlER, Suzana Pinheiro Machado. Avaliação dos títulos integrantes do programa de aquisição planificada de periódicos PAP VII. Periódicos estrangeiros para a pesquisa e o ensino das profissões agroindustriais nos cursos de pós-graduação do Brasil. Brasília [s.n], 1992b. Não publicado.
}

TABELA 5

Freqüência de uso dos títulos estrangeiros adquiridos por compra, período 1990-1999. Embrapa Amazônia Oriental. Belém, 2002

\begin{tabular}{lr}
\hline Títulos & Freqü \\
\hline Agricultural Systems & 87 \\
OCL; Oleagineux crops gras lipides ( cont. de Oleagineus) & 7 \\
Seed Science Research & 5 \\
Forest Ecology and Management & 5 \\
Biotropica & 46 \\
Agronomy Journal (cont.de Journal of the & 4 \\
American Society of Agronomy) & 4 \\
Science & 42 \\
Tropical Animal Health and Production & 42 \\
Nematologica & 42 \\
Ecology & 3 \\
Tropical Science &
\end{tabular}

(cont. de: Colonial Plant and Animal Products)

Plant Biochemical Journal

Zeitschrift fur Planzenernachrung und Bodenkunde

(cont. de: Oecologia)

Ambio-Journal of the Human Enviroment

Research and Management

Journal of Hheredity

Crop Science

Bioscience

Phytoprotection reqüência

Journal of Range Management 29

Unasylva; Revista Internacional de Silvicultura y 28

Productos Forestales

Soil science Society of America Journal

(cont. de: Soil Science Society of American Proceedings)

Soil Science and Plant Nutrition (cont.de: Soil and

Plant Food)

Agricultural and Forest Meteorology 26

Plant Physiology and Biochemistry $\quad 25$

(cont. Physiologie Vegetale)

Physiological and Molecular Plant Patholology 25

(cont. de: Physiological Plant Pathology)

Communications in Soil Science and Plant Analysis 24

Australian Journal of Agricultural Research $\quad 24$

Indian Journal of Animal Science 21

(cont. de: Indian J. of Veter.Science and Anim. Husbandry)

Australian Journal of Experimental Agriculture

(cont. de: Australian J. of Exp. Agric.and Animal Husbandy)

Interciência

Annual Review of Ecology and Systematics 20

Advances in Agronomy 20

Malaysian Agricultural Journal (cont. de: 19

Malayan AgriculturalJournal (the) e Malasyan Forester (the)

Journal of the Science of Food and Agriculture 18

Journal of the Indian Botanical Society 18

Journal of the American Chemical Society 18

Journal of Climatology 18

Indian Veterinary Journal 18

Planta Medica 16

New Zealand Journal of Technology (cont. de: New Zealand Journal of Science) 16

(continua) 
TABELA 5 (continuação)

\begin{tabular}{lc}
\hline Títulos & Freqüência \\
\hline Functional Ecology & 16 \\
Journal of Soil Science & 15 \\
Journal of Agricultural Science & 13 \\
American Journal of Botany & 13 \\
Tropical Grasslands & 12 \\
Plant Physiology & 12 \\
Journal of Applaied Ecology & 12 \\
Wood and Fiber Science (cont.de: Wood and Fiber e & 11 \\
Wood Science & \\
Weed Technology & 11 \\
New Scientist & 11 \\
Journal of Tropical Forest Science & 11 \\
Grass and Forage Science & 11 \\
(cont. de: J.of the BritishGlassland and Society) & \\
Ecological Economics & 11 \\
World Review Animal Production & 10 \\
Plant Science Bulletin & 10 \\
Madras Agricultural Journal & 10 \\
(the) (cont.de: J.of the Madras Agric. Students Union) & \\
Journal of Chemical Ecology & 10 \\
Economic Journal (the) & 10 \\
Australian Journal of Soil Research & 10 \\
\hline
\end{tabular}

Da coleção, 3.146 títulos na base Catálogo de Periódicos da Unidade (CPU), em 10 anos foram utilizados $22,63 \%$ do acervo da biblioteca e $77,36 \%$ constituiram-se, no período, como memória científica de interesse preservada em várias áreas do conhecimento.

Do total de títulos estrangeiros adquiridos por compra identificados na análise, a partir de 2000, vários tiveram as assinaturas continuadas $\mathrm{e}$ disponibilizadas na biblioteca virtual Embrapa, sendo 4,97\% na Base SDirect, 2,22\% na Ebsco, $9,40 \%$ nas bibliotecas de unidades descentralizadas, incluindo a Embrapa Amazônia Oriental, e 35,39\% na biblioteca da Sede.

Pela comparação realizada nos estudos para o Programa de Aquisição Planificada de periódicos estrangeiros para apoiar cursos de pós-graduação do MEC, a biblioteca conta com acervo e/ou facilidade de acesso à informação técnicocientífica para as atividades de P\&DI e como suporte aos três cursos de pós-graduação na região atualmente estabelecidos por contratos entre a unidade e as instituições de ensino.

tomaram emprestado e/ou citaram $74 \%$ dos títulos no campo da agronomia; $28 \%$ no campo de alimentos; $29 \%$ em medicina veterinária; $37 \%$ na engenharia agrícola; $39 \%$ na zootecnia; $57 \%$ em recursos florestais; e $45 \%$ em ecologia. Esse resultado corrobora a indicação dos títulos considerados essenciais para a pesquisa e o ensino de profissões agroindustriais, como também que os usuários da informação técnico-científica da biblioteca encontramse em sintonia com a comunidade pesquisada voltada para o ensino e a pesquisa nessas áreas do conhecimento.

\section{COMENTÁRIOS E CONCLUSÕES}

Dos 1.129 títulos identificados e analisados, 870, representando $77,05 \%$, encontram-se disponíveis na Embrapa, entre acervos, biblioteca virtual Embrapa e bases de dados, contra 259 representando 22,94\% citados na produção técnico-científica não existentes no CCPE.

Grande parte dos títulos citados não existentes no CCPE encontra-se nas zonas seis e sete, portanto não parecem representativos para efeito de aquisição.

Dos títulos emprestados/citados disponíveis na Empresa, 712, representando $81,83 \%$, foram utilizados do acervo da biblioteca da unidade, demonstrando a eficácia do acervo e periódicos de uso efetivo para as atividades de P\&DI no período, contra 102 títulos, representando 12,09\% (artigos) solicitados via comutação bibliográfica/Programa Comut, ou que podem ter sido acessados eletronicamente.
Artigo recebido em 22-07-2004 e aceito para publicação de 18 a $21 / 10 / 2004$.

\section{REFERENCIAS}

BRAGA, Ascencão. A gestão da informação. Disponivel em:_http:// www.ipv.pt/millenium/19_arq1.htm>. Acesso em: 6 maio 2004.

LANCASTER, F. W. Avaliação de periódicos. In: ___-_-_-_. Avaliação de serviços de biblioteca. Brasília : Briquet de Lemos Livros, 1996. cap. 5, p. 89-110.

MACIAS-CHAPULA, Cesar A. O papel da infometria e da cientometria e sua perspectiva nacional e internacional. Ciência da Informação, Brasília, v. 27, n. 2, p. 134-140, maio/ago. 1998.

MUEller, Suzana Pinheiro. Metodologia para avaliação de lista básica de periódicos. Ciência da Informação, Brasília, v. 20, n. 2, p. 111-118, jul./dez. 1991.

Periódicos estrangeiros prioritários para o ensino e a pesquisa da ecologia no Brasil. Ciência da Informação, Brasília, v. 21, n. 1, p. 32-39, jan./abr. 1992a.

PINHEIRO, Lena Vânia Ribeiro. Lei de Bradford: uma reformulação conceitual. Ciência da Informação, Brasília, v. 12, n. 2, p. 59-80, 1983.

$$
\text { Lei de Bradford: uma reformulação conceitual. } 1982.116 \mathrm{f} \text {. }
$$

Dissertação (Mestrado em Ciência da Informação) - Instituto Brasileiro de Informação em Ciência e Tecnologia, Universidade Federal do Rio de Janeiro, Rio de Janeiro, 1982.

PENTEADO, Roberto; QUONIAN, L. Aplicação da bibliometria na análise estratégica das competências da Embrapa. In: WOKSHOP BRASILEIRO DE INTELIGÊNCIA COMPETITIVA E GESTÃO DO CONHECIMENTO, 2., 2001. São Paulo. Inteligência competitiva: gestão do conhecimento [S.I.]; FINEP, 2001. 1 CD-ROM.

ULRICH's International periodical directory 1990-1991: classified guide to selcted list of current periodicals, foreign and domestic. 29. ed. New York : Booker, 1990, 3 v. 